\title{
EFFICACY AND SAFETY OF REPETITIVE TRANSCRANIAL MAGNETIC STIMULATION USING AN H1-COIL OR FIGURE-8-COIL IN THE TREATMENT OF UNIPOLAR MAJOR DEPRESSIVE DISORDER: A STUDY PROTOCOL FOR A RANDOMIZED CONTROLLED TRIAL
}

\author{
Igor Filipčić ${ }^{1,2,3}$, Ivona Šimunović Filipčić ${ }^{4}$, Tomislav Gajšak ${ }^{1}$, Željko Milovac ${ }^{1}$, Strahimir Sučić ${ }^{1}$, \\ Ena Ivezić ${ }^{1}$, Sandra Zečević Penić ${ }^{1}$, Ivana Orgulan ${ }^{1}$, Damir Šebo ${ }^{2}$, Vjekoslav Jeleč ${ }^{5}$ \& Žarko Bajić ${ }^{1}$

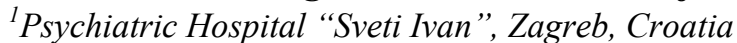 \\ ${ }^{2}$ Faculty of Dental Medicine and Health, Josip Juraj Strossmayer University of Osijek, Osijek, Croatia \\ ${ }^{3}$ School of Medicine, University of Zagreb, Zagreb, Croatia \\ ${ }^{4}$ Department of psychological medicine, University Hospital Center Zagreb, Zagreb, Croatia \\ ${ }^{5}$ Department of Neurosurgery, Clinical Hospital “Dubrava," Zagreb, Croatia
}

received: 14.10.2016;

revised: 5.5.2017;

accepted: 23.6.2017

\begin{abstract}
SUMMARY
Repetitive transcranial magnetic stimulation (rTMS) is a noninvasive technique with few side effects that has been reported to be effective in the treatment of major depressive disorder (MDD). At present, no randomized controlled trials (RCT) have compared the efficacy and safety of rTMS delivered by the figure-8-coil and the H1-coil. We describe an industry-independent, randomized, controlled, single-blinded, single-center study protocol assessing the differences in efficacy and safety of rTMS for patients diagnosed with MDD with the H1-coil and figure-8-coil as an add-on to stable pharmacotherapy or pharmacotherapy alone. Stimulation protocols follow those that led to the FDA clearance of these treatments for MDD. The sample of 76 patents in each of the three groups will be enrolled and assessed with clinical and neuropsychological tests. The primary outcome is remission rate defined as Hamilton depression rating scale (HAM-D17) score $\leq 7$ at the end of week-4.

This clinical trial will address the efficacy and safety of rTMS modalities for MDD. The evaluation of biological markers will also help to elucidate the pathophysiology of MDD and the mechanisms of action of rTMS.
\end{abstract}

Key words: repetitive transcranial magnetic stimulation - rTMS - deep TMS - dTMS - major depressive disorder

\section{INTRODUCTION}

Major depressive disorder (MDD) is a highly heterogeneous disorder that is associated with significant morbidity, disability, and mortality (Ferrari et al. 2013, GBD 2015 Disease and Injury Incidence and Prevalence Collaborators 2016, Laursen et al. 2016). Due to suboptimal treatment options and MDD's pervasiveness and common chronic and recurrent course, this disorder represents a clinical challenge concerning both acute and long-term treatment (Greden 2001, Trivedi 2006). Despite the availability of novel and effective antidepressants, it has been estimated that only $30 \%$ of patients reach the treatment goal of remission (Trivedi et al. 2006). An alternative to augmenting the antidepressant with a second medication are neuromodulation procedures, such as electroconvulsive therapy (ECT) and transcranial magnetic stimulation (TMS). While ECT is generally regarded as the most effective treatment for major depression (Anderson et al. 2008), in recent years, repetitive transcranial magnetic stimulation (rTMS) has been increasingly entering widespread use as an evidence-based treatment option (Lefaucheur et al. 2014, Milev et al. 2016).
Significant antidepressant effects of rTMS were shown in medication-free patients with treatment resistant depression compared to placebo; however, findings for augmentation (the addition of rTMS treatment to antidepressant medications) observed in randomized controlled trials (RCT) and observational studies studies are conflicting (Herwig et al. 2007, O'Reardon et al. 2007, George et al. 2010). Recently, novel forms of rTMS therapy have been investigated, such as high frequency TMS (HF-rTMS) with H1-coil over the left dorsolateral prefrontal cortex (DLPFC), which uses a different coil format that can allegedly stimulate deeper cortical and subcortical structures (Levkovitz et al. 2007, Harel et al. 2014, Levkovitz et al. 2015). This coil is designed to affect extensive neuronal pathways, including deeper cortical regions and fibers targeting subcortical regions without a significant increase of the electric field induced in superficial cortical layers (Zangen et al. 2005, Roth et al. 2007, Deng et al. 2013, Roth et al. 2014). The effectiveness of dTMS in the treatment of depression has been validated in several published studies (Levkovitz et al. 2007, Levkovitz et al. 2009, Isserles et al. 2011, Harel et al. 2014). 
Igor Filipčić, Ivona Šimunović Filipčić, Tomislav Gajšak, Željko Milovac, Strahimir Sučić, Ena Ivezić, Sandra Zečević Penić, Ivana Orgulan, Damir Šebo, Vjekoslav Jeleč \& Žarko Bajić: EFFICACY AND SAFETY OF REPETITIVE TRANSCRANIAL MAGNETIC STIMULATION USING AN H1-COIL OR FIGURE-8-COIL IN THE TREATMENT OF UNIPOLAR MAJOR DEPRESSIVE DISORDER: A STUDY PROTOCOL FOR A RANDOMIZED CONTROLLED TRIAL Psychiatria Danubina, 2018; Vol. 30, No. 1, pp 41-46

Therefore, considering the need to develop more effective strategies for the treatment of MDD, and because of the lack of large RCT investigating the acute and long-term efficacy of HF-rTMS modalities as an augmenting strategy to the antidepressants in MDD patients, the benefits and risks of different modalities have to be further evaluated. To address this issue, we will conduct an industry-independent, randomized, controlled, single-blinded, single-center study assessing the differences in efficacy and safety of HF-rTMS with the H1-coil and figure-8-coil as an add-on to pharmacotherapy or pharmacotherapy alone.

\section{Aim}

The aim of our study is to contribute to our collective knowledge regarding the treatment of depressive disorders and to fill specific gaps in our understanding of rTMS as an augmentative treatment of MDD.

\section{Primary objective}

The primary objective is the assessment of the shortterm efficacy and tolerability of HF-rTMS with the H1coil ("deep" TMS) and that with a figure-8-coil in the treatment of MDD, augmentative to the standard pharmacotherapy. Our hypothesis is that HF-rTMS with the H1-coil is more effective than a figure-8-coil HF-rTMS.

\section{Secondary objectives}

Secondary objectives are a) efficacy and safety of rTMS with a three- and six-month follow-up period, b) assessment of a three- and six-month efficacy and tolerability of HF-rTMS with H1-coil as the augmentative treatment to standard pharmacotherapy compared to the standard pharmacotherapy and psychotherapy alone, and c) Croatian sample replication of studies assessing HF-rTMS with figure-8-coil compared to the standard pharmacotherapy.

\section{Exploratory objectives}

Exploratory and supportive objectives are a) to examine whether the efficacy of rTMS is comparable across the different MDD symptoms and symptom clusters, b) to test the hypothesis that previous studies failed to detect serotonin predictive value for the rTMS treatment outcome, c) to examine whether the outcome of rTMS treatment of MDD can be predicted by baseline plasma BDNF concentration levels, d) to check whether the effects of rTMS in the treatment of MDD are moderated by chronic somatic comorbidities, e) to assess rTMS efficacy on sleep disorders in patients with MDD, f) to examine the efficacy of rTMS on hopelessness in patients diagnosed with MDD, and finally, g) to construct the multivariable model for the prediction of rTMS MDD treatment outcomes based on sociodemographic, psychological, biochemical and clinical parameters.

\section{SUBJECTS AND METHODS}

\section{Study design}

This randomized, controlled, single-blinded study will be conducted at Psychiatric Hospital "Sveti Ivan", in Zagreb, Croatia. Enrollment and concealed allocation will begin in December 2016. The study is expected to be closed by December 2017. This study is already approved by the institutional Ethics Committee and registered at ClinicalTrials.gov (NCT02917499).

The study will be conducted in accordance with the recommendations established in the Helsinki Declaration (WMA 2013). All subjects will be asked to give written informed consent for the participation, will be allowed to leave the study at any time. Data will be analyzed and published in a way that preserves anonymity of the participants.

\section{Participants}

The targeted population are patients of both gender, diagnosed with major depressive disorder. Diagnoses will be obtained by trained psychiatrists through a structured clinical interview based on DSM-5 criteria. Table 1 shows the detailed inclusion/exclusion criteria. We will choose a consecutive sample of patients, meaning that all patients who come to the hospital for any reason during the enrollment period will be assessed for eligibility by the order of their arrival. The study design will permit patients to continue pharmacotherapy during treatment with rTMS, but dose adjustment and change of the treatment will not be permitted. The cost of treatment sessions will be covered by the Croatian Health Insurance Fund as a result of positive rTMS reimbursement.

\section{Needed sample size}

During the creation of the protocol, we were not aware of any RCT comparing rTMS with H1-coil and the figure-8-coil. Therefore, the power analysis was performed for the "medium effect size" of $\mathrm{d}=0.50$. A sample size of 64 achieves $80 \%$ power at $\mathrm{p}<0.05$ to detect a standardized effect of this or larger size. To account for the expected $\leq 15 \%$ of drop out and missing data, we decided to select the sample of 76 in each of the three groups, which is the owerall sample of 228. Power analysis was performed in PASS 14 Power Analysis and Sample Size Software (2015) (NCSS, LLC, Kaysville, Utah, USA).

\section{Randomization}

Patients will be allocated to the 4-weeks HF-rTMS treatment with H1-coil, HR-rTMS treatment with figure-8-coil (with comparable standard therapy in both groups), or to the no-TMS control group treated only with the standard therapy (Figure 1). They will be randomized into three groups in 1:1:1 ratio by stratified, 
Igor Filipčić, Ivona Šimunović Filipčić, Tomislav Gajšak, Željko Milovac, Strahimir Sučić, Ena Ivezić, Sandra Zečević Penić, Ivana Orgulan, Damir Šebo, Vjekoslav Jeleč \& Žarko Bajić: EFFICACY AND SAFETY OF REPETITIVE TRANSCRANIAL MAGNETIC STIMULATION USING AN H1-COIL OR FIGURE-8-COIL IN THE TREATMENT OF UNIPOLAR MAJOR DEPRESSIVE DISORDER: A STUDY PROTOCOL FOR A RANDOMIZED CONTROLLED TRIAL Psychiatria Danubina, 2018; Vol. 30, No. 1, pp 41-46

permuted-block randomization. Stratification will be performed for age (three age groups) and gender. We will use three blocks of random sizes: 3,6 and 9. Randomization will be performed by the independent research institution (Biometrika Healthcare Research, Zagreb, Croatia), only after the successive enrollment of participants. The allocation will be concealed from the physicians and nurses who will be performing enrollment.

Table 1. Inclusion and exclusion criteria

\section{Inclusion criteria}

- Age 20-70 years

- Confirmed diagnosis of major depressive disorder

- Met standardized criteria for failure to achieve remission from antidepressant medication treatment in the current illness episode

- Psychopharmacological treatment maintained unchanged for the four weeks preceding rTMS application

- Able to read, verbalize understanding and voluntarily sign the informed consent form prior to performance of any study-specific procedures or assessments

Exclusion criteria

- Previous treatment with rTMS

- Ferromagnetic material close to the head

- Cardiac pacemaker and/or implanted electronic device

- The presence of neurological disorders (uncontrolled epilepsy, previous significant head injuries, brain surgery)

- Pregnancy or lactation

- Significant medical and/or psychiatric comorbidities

- Substance abuse in the last three months (not including caffeine or nicotine)

- Acute psychosis

- Acute suicidality

- Patient refuses to sign consent for participation in the study

Table 2. Data/instruments and the time of measurement

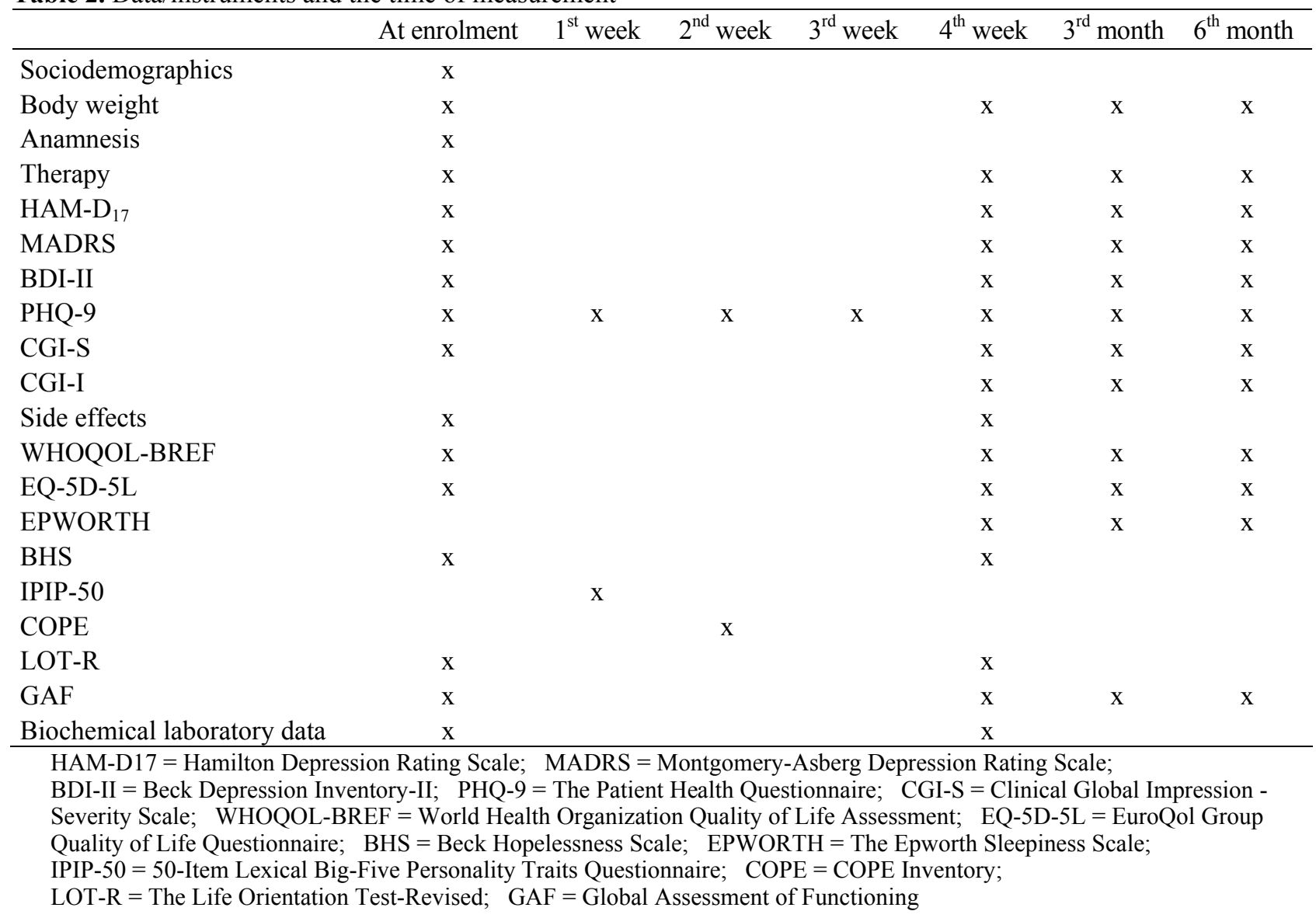




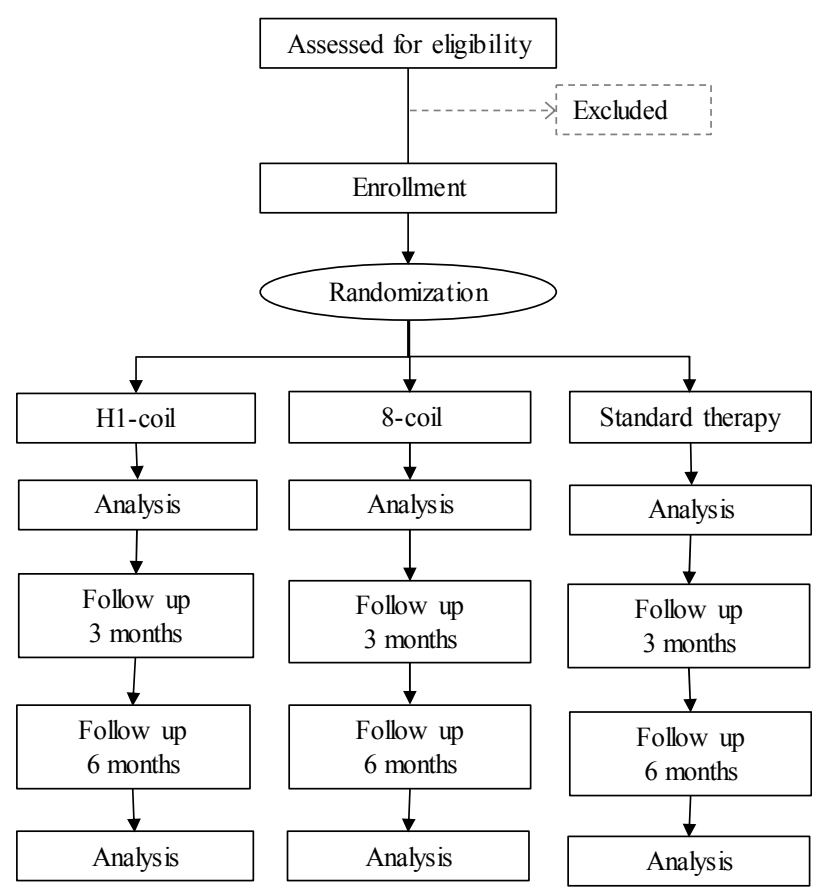

Figure 1. Study flow

\section{Intervention}

Stimulation protocol, including stimulation parameters and positioning over the left DLPFC, will follow the trails that led to FDA approval for the treatment of MDD (O'Reardon et al. 2007, Levkovitz et al. 2015) Both protocols will be administered 5 days per week for 4 weeks (total of 20 sessions). HF-rTMS with figure-8coil will be performed using a Magstim Rapid2 (Magstim Company, Spring Gardens, UK) and will include 40 minutes sessions of $10 \mathrm{~Hz}$ stimulation at $120 \%$ of motor threshold (4-seconds trains separated by 26 -seconds inter-train intervals, 75 trains totaling 3000 pulses/ session), while rTMS with the H1-coil device (Brainsway Ltd., Jerusalem, Israel) will include 20 minutes sessions of $18 \mathrm{~Hz}$ at $120 \%$ of motor threshold (2 seconds trains separated by 20 seconds inter-train intervals, 55 trains totaling 1980 pulses per session). After the enrollment, expected tolerability will be estimated based on initial mapping procedure.

\section{Outcomes}

The primary outcome is the percentage of patients who achieved remission after the 4-weeks therapy. We defined remission as Hamilton depression rating scale (HAM-D17) score $\leq 7$ (Hamilton 1960). Secondary outcomes are change in the result of HAM-D17, treatment response (HAM-D17 $\geq 50 \%$ decrease), change in the quality of life measured by WHOQOL-BREF (The WHOQOL Group 1998), safety, and tolerability of two rTMS treatment modalities. Exploratory, tertiary outcomes are change in core depression symptom severity measured by HAM-D6, change in Montgomery-Asberg Depression Rating Scale (MADRS) (Montgomery \&
Asberg 1979), remission defined by MADRS score $\leq 9$, treatment response (defined as $\geq 50 \%$ decrease in MADRS), changes in the number of patients in different HAM-D17 categories (defined as no depression $(\leq 7)$, mild (8-16), moderate (17-23) and severe $(\geq 24))$, lowering of HAM-D17 dimensions scores, change in the Pittsburgh Sleep Quality Index (Buysse et al. 1989), change in the quality of life measured by self-report WHOQOL-BREF (the WHOQOL Group 1998), safety, and tolerability. The outcomes assessment will be performed by the independent experienced psychiatrists who will be blinded for the modality of intervention. Patients will be monitored daily during the TMS sessions in order to assess safety and tolerability of the procedure, using spontaneously reported adverse events and rates of discontinuation for any of them. Patients from the control group who will be treated by standard pharmacotherapy alone will be monitored only at baseline and after 4-weeks.

\section{Possible confounders}

Possible confounders whose effect we will try to control by multivariable analysis are gender, age, education, marital status, work status, duration of MDD, diagnosis of depressive episode or the recurrent MDD, and treatment with specific antidepressants, antipsychotics, and benzodiazepines.

\section{Statistical analyses}

Analysis will be conducted in both intention-to-treat (ITT) and per-protocol (PP) populations. Missing data will be imputed as treatment failure (not achieving remission/response) or as the no-change (baseline values of continuous variables HAM-D17 and WHOQOL-BREF). The analysis of proportion of patients achieving remission will be performed by multivariable binary logistic regression. The changes in secondary outcomes (HAMD17 and WHOQOL-BREF) will be analyzed by the analysis of covariance. In all analyses, preplanned possible confounders will be included as covariates. The homogeneity of error variances across rTMS modalities will be tested by Levene's test. The homogeneity of regression slopes will be checked by testing the significance of the interaction between rTMS modality and the preplanned covariates. Partial Eta squared $\left(\eta^{2}\right)$ will be given as the standardized effect size. Sensitivity analysis of the difference between two rTMS modalities in the decrease of HAM-D17 score will be done by Quade's rANCOVA procedure. The values of baseline and aftertreatment HAM-D17 and covariates will first be ranked. Than the linear regression will be performed on the ranks of the outcome, covariates and the baseline HAMD17 scores. Finally, the Mann-Whitney test will be done using the unstandardized residuals from the previously described regression analysis as the dependent variable, and rTMS modality as the independent variable. As the standardized effect size measure for the 
Igor Filipčić, Ivona Šimunović Filipčić, Tomislav Gajšak, Željko Milovac, Strahimir Sučić, Ena Ivezić, Sandra Zečević Penić, Ivana Orgulan, Damir Šebo, Vjekoslav Jeleč \& Žarko Bajić: EFFICACY AND SAFETY OF REPETITIVE TRANSCRANIAL MAGNETIC STIMULATION USING AN H1-COIL OR FIGURE-8-COIL IN THE TREATMENT OF UNIPOLAR MAJOR DEPRESSIVE DISORDER: A STUDY PROTOCOL FOR A RANDOMIZED CONTROLLED TRIAL Psychiatria Danubina, 2018: Vol. 30, No 1 , pp 41 -46

Mann-Whitney test, $r$ will be presented. We will calculate $\mathrm{r}$ as: $\mathrm{Z} /(\mathrm{SQRT}(\mathrm{n}))$ where $\mathrm{Z}$ is a standardized $\mathrm{U}$ statistic, and $\mathrm{n}$ is the number of participants. The statistical significance of the results of sub-group analysis and sensitivity analysis of HAM-D17 score decrease will be corrected for multiple testing by sequential Holm-Bonferroni correction. In all instances, two-tailed tests will be used. The level of statistical significance will be set at $\mathrm{p}<0.05$, and we will give all confidence intervals at $95 \%$ level. Statistical data analysis will be done by NCSS 10 Statistical Software (2015) (NCSS, LLC. Kaysville, Utah, USA).

\section{Publication of research results}

Following completion of the study, a manuscript will be prepared for the primary and secondary outcomes. Additional manuscripts may be prepared to report on tertiary outcomes findings.

\section{DISCUSSION}

There is a growing body of literature demonstrating that rTMS modalities may be a safe and effective treatment for MDD. This study will be one of the largest trials to date assessing the efficacy and safety of rTMS modalities in MDD. Furthermore, this study will be first RCT assessing the efficacy and safety of adjunctive therapy between the two FDA-approved rTMS modalities, the H1-coil and the figure-8-coil. The study is adequately powered to test the primary hypothesis and to handle a study attrition of up to $15 \%$. Our treatment protocol consists of an FDA-approved protocol for both modalities; the treatment session duration in the H1-coil group will be 20 minutes compared to 40 minutes in the 8-coil group as was applied in the trails that led to FDA approval for the treatment of MDD (O'Reardon et al. 2007, Levkovitz et al. 2015). In this regard, the present study will be the first RCT assessing the difference in the antidepressant effects between these two protocols.

This clinical trial will address the efficacy and safety of HF-rTMS modalities for MDD patients with treatment-resistant depression using a randomized controlled design. Also, it will assess additive effect of add-on HFrTMS treatment to pharmacotherapy. Furthermore, we will compare the efficacy H1-coil with figure-8-coil in different MDD dimensions and symptoms to determine whether there are differences in two rTMS modalities' efficacy on different MDD dimensions and symptoms. As MDD is a multidimensional clinical entity, it is possible that we misjudged the efficacy of TMS. The evaluation of biological markers with depression response will also help to elucidate the pathophysiology of MDD and the mechanisms of action of this novel technique of neuromodulation. Therefore, our trial can generate important findings in the fields of clinical treatment of major depression and noninvasive brain stimulation.

\section{Trial status}

This trial has not completed patient recruitment at the time of submission.

\section{CONCLUSIONS}

This study will be one of the largest trials to date assessing the efficacy and safety of rTMS modalities (the H1-coil and the figure 8-coil) in MDD patients. Furthermore, this trial will be the first RCT assessing the differences between these two FDA-approved rTMS modalities (as add-on to standard pharmacotherapy) in order to provide a better understanding of rTMS as the augmentative treatment of MDD. Additionally, the evaluation of biological markers within treatment response will help to further elucidate the pathophysiology of MDD and the mechanisms of action of this novel technique of neuromodulation. Therefore, this trial could generate important findings in the fields of clinical treatment of major depression and noninvasive brain stimulation.

\section{Acknowledgements: None.}

\section{Conflict of interest: None to declare.}

\section{Contribution of individual authors:}

Igor Filipčić \& Ivona Šimunović Filipčić conceived the study.

Žarko Bajić \& Ena Ivezić designed the study protocol. Ivona Šimunović \& Žarko Bajić wrote the first draft of the manuscript.

Žarko Bajić was responsible for the statistical analyses.

Željko Milovac, Strahimir Sučić, Tomislav Gajšak, Ivana Orgulan, Sandra Zečević Penić, Damir Šebo \& Vjekoslav Jeleč contributed to critical revisions of the manuscript. All authors have read and approved the final version of the manuscript.

\section{References}

1. Anderson IM, Ferrier IN, Baldwin RC, Cowen PJ, Howard L, Lewis $G$ et al.: Evidence-based guidelines for treating depressive disorders with antidepressants: a revision of the 2000 British Association for Psychopharmacology guidelines. J Psychopharmacol 2008; 22:343-96

2. Buysse DJ, Reynolds CF, Monk TH, Berman SR, \& Kupfer DJ: The Pittsburgh Sleep Quality Index: a new instrument for psychiatric practice and research. Psychiatry Res 1989; 28:193-213

3. Deng Z-D, Lisanby $S H$ \& Peterchev AV: Electric field depth-focality tradeoff in transcranial magnetic stimulation: simulation comparison of 50 coil designs. Brain Stimul 2013; 6:1-13 
Igor Filipčić, Ivona Šimunović Filipčić, Tomislav Gajšak, Željko Milovac, Strahimir Sučić, Ena Ivezić, Sandra Zečević Penić, Ivana Orgulan, Damir Šebo, Vjekoslav Jeleč \& Žarko Bajić: EFFICACY AND SAFETY OF REPETITIVE TRANSCRANIAL MAGNETIC STIMULATION USING AN H1-COIL OR FIGURE-8-COIL IN THE TREATMENT OF UNIPOLAR MAJOR DEPRESSIVE DISORDER: A STUDY PROTOCOL FOR A RANDOMIZED CONTROLLED TRIAL Psychiatria Danubina, 2018; Vol. 30, No. 1, pp 41-46

4. Ferrari AJ, Charlson FJ, Norman RE, Patten SB, Freedman G, Murray CJL et al.: Burden of Depressive Disorders by Country, Sex, Age, and Year: Findings from the Global Burden of Disease Study 2010. PLoS Med 2013; 10:e1001547

5. GBD 2015 Disease and Injury Incidence and Prevalence Collaborators: Global, regional, and national incidence, prevalence, and years lived with disability for 310 diseases and injuries, 1990-2015: a systematic analysis for the Global Burden of Disease Study 2015. Lancet 2016; 388:1545-602

6. George MS, Lisanby SH, Avery D, McDonald WM, Durkalski $V$, Pavlicova $M$ et al.: Daily left prefrontal transcranial magnetic stimulation therapy for major depressive disorder: a sham-controlled randomized trial. Arch Gen Psychiatry 2010; 67:507-16

7. Greden JF: The burden of disease for treatment-resistant depression. J Clin Psychiatry 2001; 62:26-31

8. Hamilton $M$ : A rating scale for depression. J Neurol Neurosurg Psychiat 1960; 23:56-62

9. Harel EV, Rabany L, Deutsch L, Bloch Y, Zangen A \& Levkovitz $Y$ : $H$-coil repetitive transcranial magnetic stimulation for treatment resistant major depressive disorder: An 18-week continuation safety and feasibility study. World J Biol Psychiatry 2014; 15:298-306

10. Herwig U, Fallgatter AJ, Höppner J, Eschweiler GW, Kron M, Hajak $G$ et al.: Antidepressant effects of augmentative transcranial magnetic stimulation: randomised multicentre trial. Br J Psychiatry 2007; 191:441-8

11. Isserles M, Rosenberg O, Dannon P, Levkovitz Y, Kotler $M$, Deutsch $F$ et al.: Cognitive-emotional reactivation $d u-$ ring deep transcranial magnetic stimulation over the prefrontal cortex of depressive patients affects antidepressant outcome. J Affect Disord 2011; 128:235-42

12. Laursen TM, Musliner KL, Benros ME, Vestergaard $M \&$ Munk-Olsen T: Mortality and life expectancy in persons with severe unipolar depression. J Affect Disord 2016; 193:203-7

13. Lefaucheur J-P, André-Obadia N, Antal A, Ayache SS, Baeken $C$, Benninger DH et al.: Evidence-based guidelines on the therapeutic use of repetitive transcranial magnetic stimulation (rTMS). Clin Neurophysiol 2014, 125:2150-206

14. Levkovitz Y, Roth Y, Harel EV, Braw Y, Sheer A \& Zangen $A$ : A randomized controlled feasibility and safety study of deep transcranial magnetic stimulation. Clin Neurophysiol 2007; 118:2730-44
15. Levkovitz Y, Harel E V, Roth Y, Braw Y, Most D, Katz LN et al.: Deep transcranial magnetic stimulation over the prefrontal cortex: evaluation of antidepressant and cognitive effects in depressive patients. Brain Stimul 2009; 2:188-200

16. Levkovitz $Y$, Isserles $M$, Padberg F, Lisanby SH, Bystritsky A, Xia $G$ et al.: Efficacy and safety of deep transcranial magnetic stimulation for major depression: a prospective multicenter randomized controlled trial. World Psychiatry $2015 ; 14: 64-73$

17. Milev RV, Giacobbe P, Kennedy SH, Blumberger DM, Daskalakis ZJ, Downar J et al.: Canadian Network for Mood and Anxiety Treatments (CANMAT) 2016 Clinical Guidelines for the Management of Adults with Major Depressive Disorder: Section 4. Neurostimulation Treatments. Can J Psychiatry 2016; 61:561-75

18. Montgomery $S A$ \& Asberg $M: A$ new depression scale designed to be sensitive to change. Br J Psychiatry 1979; 134:382-9

19. O'Reardon JP, Solvason HB, Janicak PG, Sampson S, Isenberg KE, Nahas Z, et al.: Efficacy and safety of transcranial magnetic stimulation in the acute treatment of major depression: a multisite randomized controlled trial. Biol Psychiatry 2007; 62:1208-16

20. Roth Y, Amir A, Levkovitz Y \& Zangen A: Three-dimensional distribution of the electric field induced in the brain by transcranial magnetic stimulation using figure- 8 and deep H-coils. J Clin Neurophysiol 2007; 24:31-8

21. Roth Y, Pell GS, Chistyakov AV, Sinai A, Zangen A \& Zaaroor M: Motor cortex activation by $H$-coil and figure- 8 coil at different depths. Combined motor threshold and electric field distribution study. Clin Neurophysiol 2014; 125:336-43

22. The WHOQOL Group: Development of the World Health Organization WHOQOL-BREF quality of life assessment. Psychol Med 1998; 28:551-8

23. Trivedi MH: Major depressive disorder: remission of associated symptoms. J Clin Psychiatry 2006; 67:27-32

24. Trivedi MH, Rush AJ, Wisniewski SR, Nierenberg AA, Warden D, Ritz L et al.: Evaluation of outcomes with citalopram for depression using measurement-based care in $S T A R^{*} D$ : Implications for clinical practice. Am J Psychiatry 2006; 163:28-40

25. World Medical Association: World Medical Association Declaration of Helsinki: ethical principles for medical research involving human subjects. JAMA 2013; 310:2191-4

26. Zangen A, Roth $Y$, Voller $B$ \& Hallett M: Transcranial magnetic stimulation of deep brain regions: evidence for efficacy of the H-coil. Clin Neurophysiol 2005; 116:775-9

Correspondence:

Igor Filipčić, $M D, P h D$

Psychiatric Hospital "Sveti Ivan"

Jankomir 11, pp68, HR-10 090 Zagreb, Croatia

E-mail:igor.filipcic@pbsvi.hr 